\title{
Towards the brain-computer's code?
}

A simple model seems to suggest how information about an external stimulus may be gleaned from the trains of pulses neurons of the sensory systems send to the central nervous system.

Although it is generally accepted that the brain is a computer of a kind, nobody yet knows what is the computer code or codes (or there may be different codes for different purposes). The idea that memory may be encoded in the sequence of the chemical subunits of macromolecules is natural enough - there is so much memory to accommodate - but the simplest versions of it current only a few years ago cannot easily be reconciled with the interconnectedness of the neurons of the cerebral computer, or with the likelihood that there is something in the neural network view of how the brain works.

But it is also plain that such a code could not possibly apply to the way in which information is transmitted from the periphery to the central processing regions, where it seems now to be wellestablished that the activity of a neuron, like the information traffic along a neuronal channel, is measured best by the rate at which voltage pulses, or spikes, are generated within it. In due course, it will be necessary for people to be asking themselves how two such radically different coding principles are mated with each other.

Indeed, the only coded information in the brain that is, for the time being, vaguely susceptible to measurement is that from the neurons of the peripheral sensory system, but even then the meaning is far from clear. Does the rate at which a peripheral neuron "fires" provide a simple measure of the intensity of the stimulus, or of something more subtle? How is phase information embodied in the signal and - if it is not - how else can the signals from neighbouring neurons in the same sensory system be combined with each other to yield other than averages? And is the noisiness of the output of the sensory neurons simply a nuisance, or may it be more significant?

This, more or less, is the starting point for an intriguing argument about the mechanism of sensory coding in the central nervous system by André Longtin from the theoretical division at the Los Alamos National Laboratory, Adi Bulsara from the Naval Systems Center at San Diego and Frank Moss from the University of Missouri at St Louis. Interest apart, one reason for drawing attention to the article is that it appears in what must be, for most neurophysio- logists, an obscure journal: Physical Review Letters $(67,656 ; 1991)$.

The starting-point is experimental measurements of the intervals of time between successive spikes from sensory neurons stimulated by a periodic sensation. In the neurophysiology trade, it is standard practice to measure the intervals of time between successive spikes of voltage, and then to plot these as a histogram. If the stimulus is large enough, the result is much as would be expected - a peak centred on the time interval corresponding to the period of the exciting stimulus and then successively smaller peaks centred on timeintervals that are integral multiples of the underlying period. To put the point almost anthropomorphically, it is as if the excited neuron does its best to respond at intervals determined by the period of the exciting stimulus, but that it sometimes fails to do so, whereupon it will try its best to do so when the stimulus is next a maximum and, if not then, then on the next occasion and so on

Luckily, there is a little more than that to say, which is what Longtin and his colleagues attempt. They begin with data gathered more than 20 years ago from single auditory nerve fibres of the monkey and from single neurons in the primary visual cortex of the cat, each of them exposed at the periphery to a periodic stimulus. The histograms of the intervals between successive voltage spikes peak at the period of the stimulus and at integral multiples thereof. It is crucial to the argument that the intensity of the successive peaks decays exponentially, suggesting that the underlying process is stochastic in some sense.

The objective is to "construct the simplest possible physical mechanism" that captures the behaviour of the histograms. That is laudable enough, though whether it can be fairly described, as the authors describe it, as taking "a reductionist's view" of the data is another matter. Perhaps the time has come to distinguish between those who believe that minimalist models have inherent virtues and those who hold that correct models will be found to be minimalist; they might be called reductionists of the first and second kinds respectively.

That quibble apart, the minimalist model is as simple as anybody could ask. If a neuron is a two-state system, say oN or OFF, most conveniently corresponding to the condition in which the transmembrane potential is sufficient to allow the neuron to fire and that in which the membrane is hyperpolarized, as it is after an action potential has been triggered, then why not represent that two-state system by a mechanical system governed by a double-well potential? Then the question to be decided is the rate at which the mechanical system will make its way from one well to the other, which will be determined by the external stimulus (say a sinusoidal force with fixed period), the noise and the shape of the potential (notably the height of the barrier between the two wells).

Now, in principle, it is possible to calculate the behaviour of the simple model. The underlying model for the neuron shows it making transitions from one state to another, but to what do the time intervals between successive spikes of potential correspond? One possibility is that the intervals of time actually measured represent the times the system spends in one state or the other (because, since the problem is made symmetrical for simplicity, either one or the other state will give the same result). Or the measured time intervals may represent the times between successive transitions from one particular state to the other. As it happens, the mathematics yields a straightforward answer. The first interpretation should yield a histogram with peaks at every odd-integer multiple of half the underlying period, the second at integral multiples of the underlying period. It follows from that that the model entails the assumption of a "recovery" phase of some kind after the discharge of each voltage spike.

The other general finding is that of the significance of noise. If the model is anything like correct, the root mean square noise potential and the external stimulus add together arithmetically in determining the intervals between the firing of a neuron. What that means is that neuronal noise may be a way of magnifying the signal that must be interpreted with the help of the code that applies elsewhere in the nervous system. Much depends, of course, on the exact nature of the code employed in those central regions, but this could begin to qualify the general assumption that the ratio of signal to noise is a universal figure of merit for the behaviour of a communications system. John Maddox 